\title{
Incentive Schemes in Peer-to-Peer Networks
}

\author{
Oksana Loginova* Haibin $\mathrm{Lu}^{\dagger} \quad$ X. Henry Wang \\ Revised and resubmitted to \\ The B.E. Journal of Theoretical Economics
}

October 31, 2008

\begin{abstract}
In this paper we study the optimal file-sharing mechanism in a peer-to-peer network with a mechanism design perspective. This mechanism improves upon existing incentive schemes. In particular, we show that peer-approved scheme is never optimal and service-quality scheme is optimal only under certain circumstances. Moreover, we find that the optimal mechanism can be implemented by a mixture of peer-approved and service-quality schemes.
\end{abstract}

JEL Classification: D82, C72

Keywords: peer-to-peer networks, file sharing incentive schemes, mechanism design

*Department of Economics, University of Missouri-Columbia, 333 Professional Building, Columbia, MO 65211, U.S.A., e-mail: loginovao@missouri.edu (corresponding author)

${ }^{\dagger}$ Department of Computer Science, University of Missouri-Columbia, 107 Engineering Building West, Columbia, MO 65211, U.S.A., e-mail: luhaibin@missouri.edu

${ }^{\ddagger}$ Department of Economics, University of Missouri-Columbia, 318 Professional Building, Columbia, MO 65211, U.S.A., e-mail: wangx@missouri.edu 


\section{Introduction}

Peer-to-peer (P2P) networks such as Kazaa, Gnutella and BitTorrent, enable users (peers) to share their files with other users of the network. These files contain music, movies, software, etc. As concluded by Gummadi, Dunn, Saroiu, Gribble, Levy, and Zahorjan (2003), P2P file sharing now dominates the internet traffic. ${ }^{1}$ BitTorrent, the most popular network, accounts for as much as one third of the internet traffic (Pasick 2004, Livingstone 2006).

$\mathrm{P} 2 \mathrm{P}$ networks crucially depend on the willingness of peers to voluntarily share their files. Empirical observations have shown that free-riding (downloading files without sharing) is prevalent in existing P2P networks. For example, Adar and Huberman (2000) reported that nearly $70 \%$ of Gnutella users did not provide music files. ${ }^{2}$ Various incentive schemes have been proposed to motivate sharing. Two of the basic ones are peer-approved and service-quality. ${ }^{3}$ Both schemes use rating systems that assign points to each peer in the network. Rating points can be defined in different ways such as the number of files a peer shares with other peers, the number of requests the peer serves, his upload/download speed, etc. Under peer-approved scheme each peer only serves requests from peers with equal or higher ratings. In contrast, under service-quality scheme each peer serves all requests, but serves peers with higher ratings first.

While existing schemes do provide some incentives for peers to share, these schemes are ad hoc because they are not derived based on well defined objectives. In this paper we use standard mechanism design approach to find the optimal file-sharing mechanism that maximizes the total welfare, which is defined as the sum of the expected payoffs of all peers in the network. In our model the peers are divided into two rating groups. This is the simplest setup that is rich enough to allow a comparison of the optimal file-sharing mechanism with both peer-approved and servicequality incentive schemes. We show that peer-approved scheme always induces some sharing, but never yields an optimal outcome. On the other hand, service-quality scheme is optimal under certain circumstances, but can lead to a complete collapse of the network (no sharing). Remarkably, the optimal mechanism can be implemented by a mixture of peer-approved and service-quality incentive schemes.

In the following section we review the related literature. In Section 3 we introduce the model setup and present the mechanism design problem. In Section 4 we examine the performance of the existing peer-approved and service-quality schemes in our setting. We characterize the optimal mechanism in Section 5 and provide an implementation of this mechanism in Section 6. Concluding remarks appear in Section 7. Proofs of all lemmas, propositions and corollaries are provided in the appendix.

\footnotetext{
${ }^{1} \mathrm{P} 2 \mathrm{P}$ produces between $49 \%$ and $83 \%$ of the internet traffic with nighttime peaks of over $95 \%$ (Schulze and Mochalski 2007). According to another study conducted by Cisco Systems Inc (2007), at the end of 2007 P2P accounted for $51 \%$ of the internet traffic.

${ }^{2}$ See also Saroiu, Gummadi, and Steven (2002) and Hughes, Coulson, and Walkerdine (2005).

${ }^{3}$ See, for example, Ranganathan, Ripeanu, Sarin, and Foster (2003). Another basic incentive scheme is micropayment (Golle, Leyton-Brown, Mironov, and Lillibridge 2001). It rewards peers who share their files with monetary payments and charges users who download files. See the next section for more discussions on the related literature.
} 


\section{Related Literature}

A number of papers have presented extensive reviews of the existing literature on P2P networks (Krishnan, Smith, and Telang 2003, Feldman and Chuang 2005, Roczniak and Saddik 2005, Ruffo and Shifanella 2007). A central issue is providing incentives for peers to share. The main tool to address this issue in the literature is the game-theoretic approach. Two classes of incentive schemes have been considered: soft and hard schemes. The most prominent soft schemes are peer-approved and service-quality. Hard schemes, which are monetary based, include token-exchange and micropayment. Below we discuss the most relevant papers that study these schemes and their variations.

We start with soft incentive schemes. Ranganathan, Ripeanu, Sarin, and Foster (2003) and Ranganathan, Ripeanu, Sarin, and Foster (2004) were the first to use a game-theoretical approach to study peer-approved and service-quality schemes using a multi-person Prisoners' Dilemma model. ${ }^{4}$ They conclude that these schemes provide some incentives for peers to share. Many subsequent studies have examined the public good aspect of P2P networks. ${ }^{5}$ In particular, Krishnan, Smith, Tang, and Telang (2004), Antoniadis, Courcoubetis, and Mason (2004), and Antoniadis, Courcoubetis, and Strulo (2005) find that the equilibrium level of sharing is below the social optimum and propose variations of soft incentive schemes to reduce free-riding. Other papers that study soft incentive schemes include Buragohain, Agrawal, and Suri (2003), Kamvar, Schlosser, and GarciaMolina (2003), Habib and Chuang (2006), Feldman, Papadimitriou, Chuang, and Stoica (2006), and Van der Schaar and Turaga (2008).

One of the first studies that considered monetary based schemes in P2P networks is Golle, Leyton-Brown, Mironov, and Lillibridge (2001). These authors propose a micro-payment scheme that induces sharing. Other papers that examine monetary schemes include Adler, Kumar, Ross, Rubenstein, Turner, and Yao (2004) and Tan and Jarvis (2008). Recently, Huang, Huang, and Gao (2007) and Zhang, Lin, and Huai (2007) introduced schemes that combine soft and hard incentives.

Our paper is concerned with soft incentive schemes. In contrast to the above mentioned papers that use game theory to analyze specific schemes, we use mechanism design to find the optimal file sharing scheme. ${ }^{6}$ That is, we are not restricting ourselves to any particular scheme a priori. The present study complements existing literature in that it shows both peer-approved and servicequality schemes play a role in the composition of the optimal scheme. As far as we know, Shneidman and Parkes (2003) were the first to advocate that mechanism design is a natural tool to model P2P networks. Although they pointed out a number of important issues concerning mechanism design in $\mathrm{P} 2 \mathrm{P}$ networks, they did not advance any formal study. ${ }^{7}$

In order to make the model tractable, we use linear benefit and cost functions in this paper. Lin-

\footnotetext{
${ }^{4}$ These authors also study token-exchange scheme and compare it to peer-approved scheme.

${ }^{5}$ Public goods and the free-rider problem have been studied extensively in the economics literature. See, for example Groves and Ledyard (1977) and a survey by Laffont (1987).

${ }^{6}$ An exception is Antoniadis, Courcoubetis, and Mason (2004). In this paper each peer's private information is his preference parameter (type), whereas in our paper a peer's type is determined by the number of files he has. They leave out implementation details and develop an approximate solution to their mechanism design problem.

${ }^{7}$ In the context of non-P2P networks (multicast cost sharing and interdomain routing), Feigenbaum and Shenker (2002) and Feigenbaum, Papadimitriou, and Shenker (2005) have used the mechanism design approach.
} 
ear cost is the most prevalent form used in the literature (Buragohain, Agrawal, and Suri 2003, Habib and Chuang 2006, Antoniadis, Courcoubetis, and Mason 2004). While concave benefit functions are assumed in many studies, several studies adopt linear or piecewise linear benefit functions (Yu and Singh 2003, Casadesus-Masanell and Hervas-Drane 2007, Van der Schaar and Turaga 2008).

\section{Model Setup}

Consider a network with $n$ peers. Each peer has either 1 or 0 files that he can make available to other peers in the network. The number of files a peer has is private information. It is the realization of an i.i.d. random variable $\theta \in\{0,1\}$ with $\operatorname{Prob}\{\theta=1\}=\lambda$. We will refer to a peer with 1 file (0 files) as a high type (low type). We assume that the files are different.

When a peer downloads a file from another peer, he derives a benefit (net of downloading cost) that is normalized to 1 . A peer incurs cost $\gamma \in(0,1)$ when he uploads his file to another peer. Since in practice upload speed is much slower than download speed, we define a period as the amount of time needed to upload one file and assume no limit on the number of downloads within a period. ${ }^{8}$ The peers discount their future payoffs by the common factor $\delta \in(0,1) .{ }^{9}$ Hence, what matters for a peer's payoff are his discounted upload/download volumes. As an example, suppose a peer downloads 3 files in period 2 and 1 file in period 5, and uploads his file in periods 1 and 3 . His discounted download and upload volumes are $3 \delta+\delta^{4}$ and $1+\delta^{2}$, respectively. His total payoff is $3 \delta+\delta^{4}-\gamma\left(1+\delta^{2}\right)$.

There is a network designer whose objective is to maximize the sum of the peers' expected payoffs. Because the designer does not observe the realizations of the peers' types, we use mechanism design approach to solve this incomplete information game. ${ }^{10}$

Whereas a high type can imitate a low type, we assume that a low type cannot imitate a high type. By the Revelation Principle, we restrict our attention to direct mechanisms in which the peers truthfully reveal their types to the network designer. ${ }^{11}$ The game runs as follows.

(1) The designer announces an allocation rule that, for each possible vector of reported types, specifies an upload/download pattern.

(2) Each peer learns the number of files he has. Then the peers simultaneously report their types to the designer.

(3) The designer applies the allocation rule to the vector of reported types and implements the resulting upload/download pattern.

\footnotetext{
${ }^{8}$ For example, cable modem has $1.5 \mathrm{Mbps}$ download and $400 \mathrm{Kbps}$ upload, and for ADSL the corresponding speeds are $1.4 \mathrm{Mbps}$ and $400 \mathrm{Kbps}$.

${ }^{9} \mathrm{~A}$ period here may be very short ( $\delta$ is close to 1 ). The model works as long as there is some discounting. Discounting also appears in Schaar and Turaga (2008).

${ }^{10}$ See Fudenberg and Tirole (1991) and Mas-Colell, Whinston, and Green (1995) for extensive coverage of mechanism design.

${ }^{11}$ Note that the incentive constraints here are weaker than the usual ones. When there is a restriction on imitations, the Revelation Principle does not trivially apply (Green and Laffont 1986). However, in our special environment with only two types the Revelation Principle still holds.
} 
Before formulating the mechanism design problem, it is worthwhile to note that our model is static in nature. There is no entry or exit of peers and the game is over once the prescribed uploading/downloading is complete. One can think of this model as a single session of a multisession dynamic setting. In a multi-session model, peers' types can change from session to session. For example, a peer may start with no files, but can choose to share his previously downloaded file(s) in later sessions. As a result, multiple peers in a session may have the same file. The assumption made earlier that peers have different files simplifies the analysis significantly. An interesting extension of our model would be to relax this assumption and/or allow entry and exit, as noted in our concluding remarks (Section 7).

Because the peers are ex ante identical, we will focus on symmetric allocation rules, under which peers of the same type get the same treatment. Such an allocation rule can be summarized by

$$
\left\{u_{k}^{H}, d_{k}^{H}, d_{k}^{L}\right\}_{k=0}^{n} .
$$

In this triple, index $k$ keeps track of the number of peers who report high types; $u_{k}^{H}$ and $d_{k}^{H}$ are the discounted upload and download volumes of a high type, respectively; $d_{k}^{L}$ is the discounted download volume of a low type. The discounted volumes $u_{k}^{H}, d_{k}^{H}$ and $d_{k}^{L}$ are determined based on the upload/download pattern specified by the mechanism.

The following feasibility constraints will always hold in a symmetric allocation: ${ }^{12}$

$$
\begin{aligned}
& k u_{k}^{H}=k d_{k}^{H}+(n-k) d_{k}^{L}, \\
& 0 \leq u_{k}^{H} \leq \sum_{i=1}^{n-1} \delta^{i-1} \equiv \bar{u}^{H}, \\
& 0 \leq d_{k}^{H} \leq \sum_{i=1}^{k-1} \delta^{i-1} \equiv \bar{d}_{k}^{H}, \\
& 0 \leq d_{k}^{L} \leq \frac{k \sum_{i=1}^{n-k} \delta^{i-1}}{n-k} .
\end{aligned}
$$

Constraint (1) follows from the simple observation that whenever one peer is uploading a file there is another peer downloading it. Constraint (2) places the upper bound $\bar{u}^{H}$ on the discounted upload volume of a high type, $u_{k}^{H}$. Indeed, the highest discounted upload volume for a high type occurs when he uploads to the other $n-1$ peers in the first $n-1$ periods. The last two constraints are more involved. Constraint (3) stipulates that the discounted download volume of a high type, $d_{k}^{H}$, is bounded from above by $\bar{d}_{k}^{H}$. This bound is realized when each of $k$ high types uploads his file to the other $k-1$ high types in the first $k-1$ periods (in a way such that all high types receive the same discounted download volume). Similarly, constraint (4) says that the discounted download volume of a low type, $d_{k}^{L}$, is maximized when each of $k$ high types uploads his file to the $n-k$ low types in

\footnotetext{
${ }^{12}$ We implicitly assume that within one session peers do not upload files that they have downloaded from other peers. This is consistent with peers' incentives. Low-type peers have no incentive to upload since it is costly to do so. High-type peers have no incentive not to upload their own file, as the cost of uploading is the same.
} 
the first $n-k$ periods (yielding $k \sum_{i=1}^{n-k} \delta^{i-1}$ in total discounted download volume, which is equally divided among the $n-k$ low types).

Let $V_{k}^{H}$ and $V_{k}^{L}$ denote the expected payoffs of a high type and a low type, respectively, when $k$ peers report high types. Then,

$$
V_{k}^{H}=d_{k}^{H}-\gamma u_{k}^{H} \quad \text { and } \quad V_{k}^{L}=d_{k}^{L} .
$$

Consider a peer who has a file. Assuming $k$ other peers truthfully report high types, this peer's payoff is $V_{k+1}^{H}$ if he reports having one file and $V_{k}^{L}$ if he reports zero files. Because the probability of $k$ other peers being high types is $\left(\begin{array}{c}n-1 \\ k\end{array}\right) \lambda^{k}(1-\lambda)^{n-k-1}$, the peer's expected payoff from reporting truthfully is

$$
\sum_{k=0}^{n-1}\left(\begin{array}{c}
n-1 \\
k
\end{array}\right) \lambda^{k}(1-\lambda)^{n-k-1} V_{k+1}^{H},
$$

and his expected payoff from misreporting is

$$
\sum_{k=0}^{n-1}\left(\begin{array}{c}
n-1 \\
k
\end{array}\right) \lambda^{k}(1-\lambda)^{n-k-1} V_{k}^{L}
$$

Hence, the following incentive compatibility constraint induces high-type peers to report truthfully:

$$
\sum_{k=0}^{n-1}\left(\begin{array}{c}
n-1 \\
k
\end{array}\right) \lambda^{k}(1-\lambda)^{n-k-1}\left[V_{k+1}^{H}-V_{k}^{L}\right] \geq 0 .
$$

The designer of the network chooses an allocation rule $\left\{u_{k}^{H}, d_{k}^{H}, d_{k}^{L}\right\}_{k=0}^{n}$ that maximizes the sum of all peers' expected payoffs,

$$
\sum_{k=0}^{n}\left(\begin{array}{l}
n \\
k
\end{array}\right) \lambda^{k}(1-\lambda)^{n-k}\left[k V_{k}^{H}+(n-k) V_{k}^{L}\right],
$$

subject to the feasibility constraints (1) through (4) and the incentive compatibility constraint (5). We will refer to this problem as the mechanism design problem (MD).

Using the equality constraint (1), the optimization problem (MD) can be rewritten in terms of 
$u_{k}^{H}$ and $d_{k}^{H}$ only:

$$
\begin{aligned}
& \max _{\left\{u_{k}^{H}, d_{k}^{H}\right\}_{k=0}^{n}} \quad(1-\gamma) \sum_{k=0}^{n}\left(\begin{array}{c}
n \\
k
\end{array}\right) \lambda^{k}(1-\lambda)^{n-k} k u_{k}^{H} \\
& \text { s.t. } \quad 0 \leq u_{k}^{H} \leq \bar{u}^{H}, \quad 0 \leq d_{k}^{H} \leq \bar{d}_{k}^{H}, \\
& \quad 0 \leq u_{k}^{H}-d_{k}^{H} \leq \sum_{i=1}^{n-k} \delta^{i-1}, \quad \text { and } \\
& \sum_{k=0}^{n-1}\left(\begin{array}{c}
n-1 \\
k
\end{array}\right) \lambda^{k}(1-\lambda)^{n-k-1}\left[d_{k+1}^{H}-\gamma u_{k+1}^{H}-\frac{k}{n-k}\left(u_{k}^{H}-d_{k}^{H}\right)\right] \geq 0 .
\end{aligned}
$$

We will refer to this reduced mechanism design problem as $\left(\mathrm{MD}^{\prime}\right)$. Given a solution $\left\{\hat{u}_{k}^{H}, \hat{d}_{k}^{H}\right\}_{k=0}^{n}$ to $\left(\mathrm{MD}^{\prime}\right)$, the corresponding solution to the original problem (MD) is $\left\{\hat{u}_{k}^{H}, \hat{d}_{k}^{H}, \hat{d}_{k}^{L}\right\}_{k=0}^{n}$, where

$$
\hat{d}_{k}^{L}=\frac{k}{n-k}\left(\hat{u}_{k}^{H}-\hat{d}_{k}^{H}\right)
$$

Before we investigate the solution to $\left(\mathrm{MD}^{\prime}\right)$, we examine the existing service-quality and peer approved incentive schemes in the above framework.

\section{Service-Quality and Peer-Approved Schemes}

First, imagine that the network designer observes the peers' types. The unconstrained optimum requires each high type to upload his file to every other peer for $n-1$ consecutive periods. That is, $u_{k}^{H}$ is set to its upper bound $\bar{u}^{H}$.

Now suppose we are in the environment with private information. Does the unconstrained optimum satisfy the incentive compatibility constraint (8)? Observe that higher values of $d_{k}^{H}$ increase the left-hand side of (8) without affecting the objective function (6). Hence, the best hope is to make each high type to upload to all other high types first and then serve the low types. That is, both $d_{k}^{H}$ and $u_{k}^{H}$ are set to their upper bounds $\bar{d}_{k}^{H}$ and $\bar{u}^{H}$, respectively. This is identical to service-quality incentive scheme, under which each peer serves all requests in an order according to their ratings. Indeed, the peers are naturally divided into two rating groups. The high-rating group consists of those peers who reported having one file and the low-rating group consists of those who have no files or choose not to share. Hence, service-quality scheme requires a peer who reports one file to first upload his file to all other peers in the high-rating group, and then to the rest of the peers. ${ }^{13}$ In symbols,

$$
u_{k}^{H, S Q}=\bar{u}^{H} \quad \text { and } \quad d_{k}^{H, S Q}=\bar{d}_{k}^{H},
$$

where the superscript "SQ" denotes discounted upload/download volumes under service-quality

\footnotetext{
${ }^{13}$ Note that discounting is the means of differentiating payoffs between the two rating groups in quality-service scheme.
} 
scheme.

Because a high-type peer has two choices: report zero files or report one file, and a low-type peer can only report zero files, there are two possible symmetric Nash equilibria (NE). In the no-sharing $\mathrm{NE}$ all high types report zero files, i.e., for a high type reporting zero files is optimal if all other high types report no files. In the sharing NE all high types report one file. That is, for a high type reporting one file is optimal if all other high types report one file. ${ }^{14}$

To see whether sharing is a NE under service-quality scheme, we evaluate the incentive compatibility constraint (8) at $u_{k}^{H, S Q}$ and $d_{k}^{H, S Q}$. This yields

$$
\sum_{k=0}^{n-1}\left(\begin{array}{c}
n-1 \\
k
\end{array}\right) \lambda^{k}(1-\lambda)^{n-k-1}\left[\bar{d}_{k+1}^{H}-\gamma \bar{u}^{H}-\frac{k}{n-k}\left(\bar{u}^{H}-\bar{d}_{k}^{H}\right)\right] \geq 0 .
$$

Applying the fact that $\sum_{k=0}^{n-1}\left(\begin{array}{c}n-1 \\ k\end{array}\right) \lambda^{k}(1-\lambda)^{n-k-1}=1$, the above inequality becomes

$$
\gamma \bar{u}^{H} \leq \sum_{k=0}^{n-1}\left(\begin{array}{c}
n-1 \\
k
\end{array}\right) \lambda^{k}(1-\lambda)^{n-k-1}\left[\bar{d}_{k+1}^{H}-\frac{k}{n-k}\left(\bar{u}^{H}-\bar{d}_{k}^{H}\right)\right] .
$$

Dividing both sides of (9) by $\bar{u}^{H}$, substituting $\bar{u}^{H}$ and $\bar{d}_{k}^{H}$ by their defining expressions in (2) and (3), and simplifying (see the appendix for the derivation) yield

$$
\gamma \leq \frac{1-\lambda+\delta^{n-1} \lambda-(1-\lambda+\delta \lambda)^{n-1}}{\left(1-\delta^{n-1}\right)(1-\lambda)}
$$

It follows that sharing is a NE under service-quality scheme if and only if $(\star)$ holds. This inequality deserves a discussion. Because of discounting and the fact that peers in the high-rating group download before those in the low-rating group, the gross benefit for a peer in the high-rating group is higher than that in the low-rating group from downloading later. When making his choice, a high-type peer compares the benefit net of the uploading costs from joining the high-rating group and the benefit from joining the low-rating group. A larger fraction of high-type peers $(\lambda)$ increases the gap between the benefits of the two groups without affecting the uploading costs. A smaller uploading cost parameter $(\gamma)$ decreases the uploading costs. Hence, the incentive to join the highrating group is stronger for larger values of $\lambda$ and smaller values of $\gamma$.

Regarding the effect of the discount factor $(\delta)$, a lower $\delta$ decreases the benefits of both groups and also the uploading costs. The negative effect on the benefit of the low-rating group is more pronounced. This combined with the fact that the decrease in benefit of the high-rating group is offset by the decrease in the uploading costs makes the high-rating group more appealing. It follows that the incentive to join the high-rating group is stronger for smaller values of $\delta$.

The above intuitions are confirmed by the next lemma, which shows that $(\star)$ is more likely to be satisfied the smaller is the uploading cost parameter, the larger is the fraction of peers who have

\footnotetext{
${ }^{14}$ Note that under service-quality scheme no-sharing is a NE. This also applies to peer-approved scheme discussed below.
} 
files, and the smaller is the discounting factor.

Lemma 1. Inequality $(\star)$ is satisfied if $\gamma$ is small, $\lambda$ is large, or $\delta$ is small.

The corresponding total equilibrium expected payoff under service-quality is

$$
Z^{S Q}= \begin{cases}(1-\gamma) \sum_{k=0}^{n}\left(\begin{array}{l}
n \\
k
\end{array}\right) \lambda^{k}(1-\lambda)^{n-k} k \bar{u}^{H}, & \text { if }(\star) \text { holds } \\
0, & \text { if otherwise }\end{cases}
$$

Next, we examine peer-approved incentive scheme. Under peer-approved scheme each peer only serves requests from peers with equal or higher ratings. In our framework, peer-approved scheme requires peers in the high-rating group only upload to each other (peers in the low-rating group will get no files). In symbols,

$$
u_{k}^{H, P A}=d_{k}^{H, P A}=\bar{d}_{k}^{H},
$$

where the superscript "PA" denotes discounted upload/download volumes under peer-approved scheme.

It is easy to see that the incentive compatibility constraint (8) is satisfied at $u_{k}^{H, P A}$ and $d_{k}^{H, P A}$. Hence, sharing is a NE under peer-approved scheme. The corresponding total equilibrium expected payoff is

$$
Z^{P A}=(1-\gamma) \sum_{k=0}^{n}\left(\begin{array}{l}
n \\
k
\end{array}\right) \lambda^{k}(1-\lambda)^{n-k} k \bar{d}_{k}^{H} .
$$

Comparing the two incentive schemes, we see that when $(\star)$ holds, sharing occurs under both schemes but more uploading/downloading takes place under service-quality scheme. When $(\star)$ does not hold, sharing occurs under peer-approved scheme, but not under service-quality scheme.

\section{Optimal Mechanism}

In this section we characterize the optimal mechanism and then compare it with service-quality and peer-approved incentives schemes.

Because higher values of $d_{k}^{H}$ do not affect the objective (6) but increase the left-hand side of the incentive compatibility constraint (8), we can restrict our attention to solutions $\left\{\hat{u}_{k}^{H}, \hat{d}_{k}^{H}\right\}_{k=0}^{n}$ in which $\hat{d}_{k}^{H}=\bar{d}_{k}^{H}$. A potential candidate for $\hat{u}_{k}^{H}$ is its upper bound $\bar{u}^{H}$, as in service-quality scheme. Obviously, $\left\{\bar{u}^{H}, \bar{d}_{k}^{H}\right\}_{k=0}^{n}$ is the solution to $\left(\mathrm{MD}^{\prime}\right)$ if $(\star)$ holds.

If $(\star)$ does not hold, (8) becomes binding and $\hat{u}_{k}^{H}$ is a solution to

$$
\begin{aligned}
\max _{\left\{u_{k}^{H}\right\}_{k=0}^{n}} & (1-\gamma) \sum_{k=0}^{n}\left(\begin{array}{c}
n \\
k
\end{array}\right) \lambda^{k}(1-\lambda)^{n-k} k u_{k}^{H} \\
\text { s.t. } & \bar{d}_{k}^{H} \leq u_{k}^{H} \leq \bar{u}^{H} \\
& \sum_{k=0}^{n-1}\left(\begin{array}{c}
n-1 \\
k
\end{array}\right) \lambda^{k}(1-\lambda)^{n-k-1}\left[\bar{d}_{k+1}^{H}-\gamma u_{k+1}^{H}-\frac{k}{n-k}\left(u_{k}^{H}-\bar{d}_{k}^{H}\right)\right]=0 .
\end{aligned}
$$


We will refer to this problem as (P). The next lemma shows that this problem has multiple solutions.

Lemma 2. Suppose $(\star)$ does not hold. Then any $\left\{u_{k}^{H}\right\}_{k=0}^{n}$ that satisfies constraints (12) and (13) is a solution to $(P)$.

The next proposition states that the optimal mechanism allows the high-type peers to download to their maximum levels but penalizes the low-type peers by sometimes limiting their download volumes.

Proposition 1. The mechanism design problem $\left(M D^{\prime}\right)$ has

(i) a unique solution given by $\hat{u}_{k}^{H}=\bar{u}^{H}$ and $\hat{d}_{k}^{H}=\bar{d}_{k}^{H}$ if (*) holds;

(ii) a continuum of solutions satisfying

$$
\begin{aligned}
& \hat{d}_{k}^{H}=\bar{d}_{k}^{H}, \quad \bar{d}_{k}^{H} \leq \hat{u}_{k}^{H} \leq \bar{u}^{H}, \quad \text { and } \\
& \sum_{k=0}^{n-1}\left(\begin{array}{c}
n-1 \\
k
\end{array}\right) \lambda^{k}(1-\lambda)^{n-k-1} \hat{u}_{k+1}^{H}=\frac{1-(1+\lambda+\delta \lambda)^{n-1}}{(\gamma+\lambda-\gamma \lambda)(1-\delta)}
\end{aligned}
$$

if $(\star)$ does not hold.

Mathematically, $\left(\mathrm{MD}^{\prime}\right)$ is a linear programming problem with a linear objective and linear constraints. The optimal solution is either a point or a convex set. The former corresponds to part (i), while the latter corresponds to part (ii) of Proposition 1.

Substituting the solution(s) into the designer's objective function yields the following result.

Lemma 3. Under the optimal mechanism, the total expected payoff is

$$
\hat{Z} \equiv \begin{cases}(1-\gamma) \sum_{k=0}^{n}\left(\begin{array}{l}
n \\
k
\end{array}\right) \lambda^{k}(1-\lambda)^{n-k} k \bar{u}^{H}, & \text { if }(\star) \text { holds } \\
\frac{1-\gamma}{\gamma+\lambda-\gamma \lambda} \sum_{k=0}^{n}\left(\begin{array}{l}
n \\
k
\end{array}\right) \lambda^{k}(1-\lambda)^{n-k} k \bar{d}_{k}^{H}, & \text { if otherwise. }\end{cases}
$$

It is straightforward to verify that the two values of $\hat{Z}$ in $(14)$ are equal when $(\star)$ holds as an equality, as expected because of continuity. Contrasting (10) with (14) we see that $(\star)$ is critical for quality-service scheme, but not for the optimal mechanism. If $(\star)$ does not hold, service-quality scheme breaks down completely. However, the optimal mechanism continues to produce an outcome with sharing.

The next proposition compares the optimal mechanism with service-quality and peer-approved incentive schemes.

Proposition 2. The following relationships hold regarding the total expected payoffs under peerapproved scheme, service-quality scheme and the optimal mechanism:

(i) if ( $\star$ ) holds then $\hat{Z}=Z^{S Q}>Z^{P A}>0$;

(ii) if ( $\star$ ) does not hold then $\hat{Z}>Z^{P A}>Z^{S Q}=0$. 
From this proposition, we conclude the following. Service-quality scheme is optimal under certain parameter configurations, but can lead to the worst possible outcome. On the other hand, peerapproved scheme always induces some sharing, but is never optimal.

\section{Implementation of Optimal Mechanism}

The optimal mechanism obtained in Section 5 can be implemented by a mixture of peer-approved and service-quality incentive schemes. Let $\alpha$ denote a number from the unit interval (i.e., $\alpha \in[0,1]$ ). Here is the scheme we propose. Suppose $k$ peers report high types. Each of these peers will first upload his file to the other $k-1$ high-type peers in the first $k-1$ periods. Next, he serves $j$ out of the remaining $n-k$ peers in periods $k$ through $k+j-1$, where $j$ is a random variable with the Binomial distribution $\operatorname{Bin}(n-k, \alpha)$. In other words, he uploads independently to each of these $n-k$ peers with probability $\alpha$. This symmetric scheme is summarized by

$$
\left\{u_{k}^{H}(\alpha), d_{k}^{H}(\alpha), d_{k}^{L}(\alpha)\right\}_{k=0}^{n},
$$

where

$$
\begin{aligned}
& u_{k}^{H}(\alpha)=\bar{d}_{k}^{H}+\sum_{j=0}^{n-k}\left[\left(\begin{array}{c}
n-k \\
j
\end{array}\right) \alpha^{j}(1-\alpha)^{n-k-j} \sum_{l=k}^{k+j-1} \delta^{l-1}\right], \\
& d_{k}^{H}(\alpha)=\bar{d}_{k}^{H}, \quad \text { and } \\
& d_{k}^{L}(\alpha)=\frac{k}{n-k} \sum_{j=0}^{n-k}\left[\left(\begin{array}{c}
n-k \\
j
\end{array}\right) \alpha^{j}(1-\alpha)^{n-k-j} \sum_{l=k}^{k+j-1} \delta^{l-1}\right] .
\end{aligned}
$$

Note that the above scheme becomes peer-approved when $\alpha=0$, it becomes service-quality when $\alpha=1$. The following proposition shows that this scheme implements the optimal mechanism for an appropriately chosen $\alpha$.

Proposition 3. There exists a unique $\hat{\alpha}$ such that $\left\{u_{k}^{H}(\hat{\alpha}), d_{k}^{H}(\hat{\alpha}), d_{k}^{L}(\hat{\alpha})\right\}_{k=0}^{n}$ implements an optimal solution to $\left(M D^{\prime}\right)$. Moreover, if $(\star)$ holds then $\hat{\alpha}=1$ and if $(\star)$ does not hold then $\hat{\alpha} \in(0,1)$.

This proposition implies that if $(\star)$ holds, the proposed scheme $\left\{u_{k}^{H}(\hat{\alpha}), d_{k}^{H}(\hat{\alpha}), d_{k}^{L}(\hat{\alpha})\right\}_{k=0}^{n}$ corresponds to service-quality scheme. If $(\star)$ does not hold, it is a non-trivial mixture of peer-approved and service-quality schemes. In the latter case, while peer-approved and service-quality schemes alone do not implement the optimal mechanism, remarkably, the mixture does.

\section{Concluding Remarks}

In this paper we used mechanism design approach to find the optimal file-sharing mechanism in a P2P network, which improves upon existing incentive schemes. In particular, we showed that peer-approved scheme is never optimal and service-quality scheme is optimal only under certain 
circumstances. Moreover, we found that the optimal mechanism can be implemented by a mixture of peer-approved and service-quality schemes.

The framework we adopted in this paper is simple and static in nature. Much more remains to be investigated. One direction is to introduce heterogeneity into the model. For example, peers may be endowed with more than one file, and/or the files may have different values. Also, peers might have different upload/download bandwidths, hence take different amounts of time to complete uploading and downloading of a file. Another direction is to introduce dynamics. In particular, a peer should be able to share not only his original file but also the files he received from others. Entry and exit of peers may also be allowed in a dynamic model. A third direction is to explore whether our linear benefit and cost functions can be generalized to allow for other functional forms, such as concave benefit and/or convex cost functions. 


\section{Appendix}

Derivation of ( $\star$ ): Dividing both sides of (9) by $\bar{u}^{H}$ and substituting $\bar{u}^{H}$ and $\bar{d}_{k}^{H}$ by their defining expressions in (2) and (3) yield

$$
\gamma \leq \sum_{k=0}^{n-1}\left(\begin{array}{c}
n-1 \\
k
\end{array}\right) \lambda^{k}(1-\lambda)^{n-k-1}\left[\frac{\sum_{i=1}^{k} \delta^{i-1}}{\sum_{i=1}^{n-1} \delta^{i-1}}-\frac{k}{n-k}\left(1-\frac{\sum_{i=1}^{k-1} \delta^{i-1}}{\sum_{i=1}^{n-1} \delta^{i-1}}\right)\right] .
$$

Applying the geometric series formula to $\sum_{i=1}^{k} \delta^{i-1}, \sum_{i=1}^{k-1} \delta^{i-1}$, and $\sum_{i=1}^{n-1} \delta^{i-1}$ in the above inequality yields

$$
\gamma \leq \sum_{k=0}^{n-1}\left(\begin{array}{c}
n-1 \\
k
\end{array}\right) \lambda^{k}(1-\lambda)^{n-k-1}\left[\frac{1-\delta^{k}}{1-\delta^{n-1}}-\frac{k}{n-k}\left(1-\frac{1-\delta^{k-1}}{1-\delta^{n-1}}\right)\right]
$$

or

$$
\begin{aligned}
& \gamma\left(1-\delta^{n-1}\right) \leq \sum_{k=0}^{n-1}\left(\begin{array}{c}
n-1 \\
k
\end{array}\right) \lambda^{k}(1-\lambda)^{n-k-1}\left[1-\delta^{k}-\frac{k}{n-k}\left(\delta^{k-1}-\delta^{n-1}\right)\right] \\
& =\sum_{k=0}^{n-1}\left(\begin{array}{c}
n-1 \\
k
\end{array}\right) \lambda^{k}(1-\lambda)^{n-k-1}\left(1-\delta^{k}\right)-\sum_{k=1}^{n} \frac{k}{n-k}\left(\begin{array}{c}
n-1 \\
k
\end{array}\right) \lambda^{k}(1-\lambda)^{n-k-1}\left(\delta^{k-1}-\delta^{n-1}\right) .
\end{aligned}
$$

Because

$$
\frac{k}{n-k}\left(\begin{array}{c}
n-1 \\
k
\end{array}\right)=\left(\begin{array}{l}
n-1 \\
k-1
\end{array}\right)
$$

the right-hand side of (15) becomes

$$
\sum_{k=0}^{n-1}\left(\begin{array}{c}
n-1 \\
k
\end{array}\right) \lambda^{k}(1-\lambda)^{n-k-1}\left(1-\delta^{k}\right)-\sum_{k=1}^{n}\left(\begin{array}{c}
n-1 \\
k-1
\end{array}\right) \lambda^{k}(1-\lambda)^{n-k-1}\left(\delta^{k-1}-\delta^{n-1}\right) .
$$

Applying the binomial formula to the first term in (16) yields

$$
\sum_{k=0}^{n-1}\left(\begin{array}{c}
n-1 \\
k
\end{array}\right) \lambda^{k}(1-\lambda)^{n-k-1}\left(1-\delta^{k}\right)=1-(1-\lambda+\delta \lambda)^{n-1} .
$$

The second term in (16) can also be simplified:

$$
\begin{aligned}
& \sum_{k=1}^{n}\left(\begin{array}{l}
n-1 \\
k-1
\end{array}\right) \lambda^{k}(1-\lambda)^{n-k-1}\left(\delta^{k-1}-\delta^{n-1}\right)=\sum_{k=0}^{n-1}\left(\begin{array}{c}
n-1 \\
k
\end{array}\right) \lambda^{k+1}(1-\lambda)^{n-k-2}\left(\delta^{k}-\delta^{n-1}\right) \\
& =\frac{\lambda}{1-\lambda} \sum_{k=0}^{n-1}\left(\begin{array}{c}
n-1 \\
k
\end{array}\right) \lambda^{k}(1-\lambda)^{n-k-1}\left(\delta^{k}-\delta^{n-1}\right)=\frac{\lambda}{1-\lambda}\left((1-\lambda+\delta \lambda)^{n-1}-\delta^{n-1}\right) .
\end{aligned}
$$


Hence, (15) can be rewritten as

$$
\gamma\left(1-\delta^{n-1}\right) \leq 1-(1-\lambda+\delta \lambda)^{n-1}-\frac{\lambda}{1-\lambda}\left[(1-\lambda+\delta \lambda)^{n-1}-\delta^{n-1}\right],
$$

from which $(\star)$ follows immediately.

Proof of Lemma 1: Let

$$
f(\lambda, \delta) \equiv \frac{1-\lambda+\delta^{n-1} \lambda-(1-\lambda+\delta \lambda)^{n-1}}{\left(1-\delta^{n-1}\right)(1-\lambda)}
$$

denote the right-hand side of $(\star)$. Below we will show that $f(\lambda, \delta)$ increases in $\lambda$ and decreases in $\delta$. These properties of $f(\lambda, \delta)$ together with the obvious fact that $(\star)$ holds for $\gamma=0, \lambda=1$, or $\delta=0$ prove the lemma. Differentiating $f(\lambda, \delta)$ with respect to $\lambda$ yields

$$
\frac{\partial}{\partial \lambda} f(\lambda, \delta)=\frac{(1-\lambda)(1-\delta)(n-1)(1-\lambda+\delta \lambda)^{n-2}-\left[(1-\lambda+\delta \lambda)^{n-1}-\delta^{n-1}\right]}{\left(1-\delta^{n-1}\right)(1-\lambda)^{2}} .
$$

Substituting

$$
(1-\lambda+\delta \lambda)^{n-1}-\delta^{n-1}=(1-\lambda)(1-\delta) \sum_{k=0}^{n-2}(1-\lambda+\delta \lambda)^{n-2-k} \delta^{k}
$$

into (17) yields

$$
\begin{aligned}
\frac{\partial}{\partial \lambda} f(\lambda, \delta) & =\frac{1-\delta}{\left(1-\delta^{n-1}\right)(1-\lambda)}\left[(n-1)(1-\lambda+\delta \lambda)^{n-2}-\sum_{k=0}^{n-2}(1-\lambda+\delta \lambda)^{n-2-k} \delta^{k}\right] \\
& =\frac{1-\delta}{\left(1-\delta^{n-1}\right)(1-\lambda)} \sum_{k=0}^{n-2}\left[(1-\lambda+\delta \lambda)^{n-2}-(1-\lambda+\delta \lambda)^{n-2-k} \delta^{k}\right] \\
& =\frac{(1-\delta)(1-\lambda+\delta \lambda)^{n-2}}{\left(1-\delta^{n-1}\right)(1-\lambda)} \sum_{k=0}^{n-2}\left[1-\left(\frac{\delta}{1-\lambda+\delta \lambda}\right)^{k}\right] .
\end{aligned}
$$

Because $(1-\delta)(1-\lambda)>0$, or equivalently $1-\lambda+\delta \lambda>\delta$,

$$
\sum_{k=0}^{n-2}\left[1-\left(\frac{\delta}{1-\lambda+\delta \lambda}\right)^{k}\right]>0
$$

It follows $\partial f(\lambda, \delta) / \partial \lambda>0$. Differentiating $f(\lambda, \delta)$ with respect to $\delta$ yields

$$
\frac{\partial}{\partial \delta} f(\lambda, \delta)=-\frac{(n-1)\left[\lambda(1-\lambda+\delta \lambda)^{n-2}\left(1-\delta^{n-1}\right)-\delta^{n-2}\left(1-(1-\lambda+\delta \lambda)^{n-1}\right)\right]}{\left(1-\delta^{n-1}\right)^{2}(1-\lambda)} .
$$

Substituting

$$
1-\delta^{n-1}=(1-\delta) \sum_{k=0}^{n-2} \delta^{k}
$$


and

$$
1-(1-\lambda+\delta \lambda)^{n-1}=\lambda(1-\delta) \sum_{k=0}^{n-2}(1-\lambda+\delta \lambda)^{k}
$$

into (18) yields

$$
\begin{aligned}
\frac{\partial}{\partial \delta} f(\lambda, \delta) & =-\frac{(n-1)(1-\delta) \lambda}{\left(1-\delta^{n-1}\right)^{2}(1-\lambda)} \sum_{k=0}^{n-2}\left[(1-\lambda+\delta \lambda)^{n-2} \delta^{k}-\delta^{n-2}(1-\lambda+\delta \lambda)^{k}\right] \\
& =-\frac{(n-1)(1-\delta) \lambda(1-\lambda+\delta \lambda)^{n-2}}{\left(1-\delta^{n-1}\right)^{2}(1-\lambda)} \sum_{k=0}^{n-2} \delta^{k}\left[1-\left(\frac{\delta}{1-\lambda+\delta \lambda}\right)^{n-2-k}\right] .
\end{aligned}
$$

Because $1-\lambda+\delta \lambda>\delta$,

$$
\sum_{k=0}^{n-2} \delta^{k}\left[1-\left(\frac{\delta}{1-\lambda+\delta \lambda}\right)^{n-2-k}\right]>0
$$

which implies $\partial f(\lambda, \delta) / \partial \delta<0$.

Proof of Lemma 2: Because

$$
k\left(\begin{array}{l}
n \\
k
\end{array}\right)=n\left(\begin{array}{l}
n-1 \\
k-1
\end{array}\right)
$$

the objective of $(\mathrm{P})$ can be rewritten as

$$
\begin{gathered}
(1-\gamma) \sum_{k=1}^{n}\left(\begin{array}{l}
n \\
k
\end{array}\right) \lambda^{k}(1-\lambda)^{n-k} k u_{k}^{H}=(1-\gamma) n \sum_{k=1}^{n}\left(\begin{array}{c}
n-1 \\
k-1
\end{array}\right) \lambda^{k}(1-\lambda)^{n-k} u_{k}^{H} \\
=(1-\gamma) \lambda n \sum_{k=0}^{n-1}\left(\begin{array}{c}
n-1 \\
k
\end{array}\right) \lambda^{k}(1-\lambda)^{n-k-1} u_{k+1}^{H} .
\end{gathered}
$$

Next, (13) can be rewritten as

$$
\begin{gathered}
\sum_{k=0}^{n-1}\left(\begin{array}{c}
n-1 \\
k
\end{array}\right) \lambda^{k}(1-\lambda)^{n-k-1}\left(\bar{d}_{k+1}^{H}-\gamma u_{k+1}^{H}\right)=\sum_{k=1}^{n-1} \frac{k}{n-k}\left(\begin{array}{c}
n-1 \\
k
\end{array}\right) \lambda^{k}(1-\lambda)^{n-k-1}\left(u_{k}^{H}-\bar{d}_{k}^{H}\right), \\
\sum_{k=0}^{n-1}\left(\begin{array}{c}
n-1 \\
k
\end{array}\right) \lambda^{k}(1-\lambda)^{n-k-1}\left(\bar{d}_{k+1}^{H}-\gamma u_{k+1}^{H}\right)=\sum_{k=1}^{n-1}\left(\begin{array}{c}
n-1 \\
k-1
\end{array}\right) \lambda^{k}(1-\lambda)^{n-k-1}\left(u_{k}^{H}-\bar{d}_{k}^{H}\right),
\end{gathered}
$$

or

$$
\sum_{k=0}^{n-1}\left(\begin{array}{c}
n-1 \\
k
\end{array}\right) \lambda^{k}(1-\lambda)^{n-k-1}\left(\bar{d}_{k+1}^{H}-\gamma u_{k+1}^{H}\right)=\sum_{k=0}^{n-2}\left(\begin{array}{c}
n-1 \\
k
\end{array}\right) \lambda^{k+1}(1-\lambda)^{n-k-2}\left(u_{k+1}^{H}-\bar{d}_{k+1}^{H}\right) .
$$

Note that $\bar{d}_{n}^{H}=\bar{u}^{H}$. It follows from (12) that $u_{n}^{H}=\bar{u}^{H}$. Adding $\lambda^{n}(1-\lambda)^{-1}\left(u_{n}^{H}-\bar{d}_{n}^{H}\right)$ to the 
right-hand side of the above equality leads to

$$
\sum_{k=0}^{n-1}\left(\begin{array}{c}
n-1 \\
k
\end{array}\right) \lambda^{k}(1-\lambda)^{n-k-1}\left(\bar{d}_{k+1}^{H}-\gamma u_{k+1}^{H}\right)=\frac{\lambda}{1-\lambda} \sum_{k=0}^{n-1}\left(\begin{array}{c}
n-1 \\
k
\end{array}\right) \lambda^{k}(1-\lambda)^{n-k-1}\left(u_{k+1}^{H}-\bar{d}_{k+1}^{H}\right),
$$

or

$$
\sum_{k=0}^{n-1}\left(\begin{array}{c}
n-1 \\
k
\end{array}\right) \lambda^{k}(1-\lambda)^{n-k-1} u_{k+1}^{H}=\frac{1}{\gamma+\lambda-\gamma \lambda} \sum_{k=0}^{n-1}\left(\begin{array}{c}
n-1 \\
k
\end{array}\right) \lambda^{k}(1-\lambda)^{n-k-1} \bar{d}_{k+1}^{H} .
$$

Substituting

$$
\bar{d}_{k+1}^{H}=\frac{1-\delta^{k}}{1-\delta}
$$

into (20) yields

$$
\sum_{k=0}^{n-1}\left(\begin{array}{c}
n-1 \\
k
\end{array}\right) \lambda^{k}(1-\lambda)^{n-k-1} u_{k+1}^{H}=\frac{1-(1-\lambda+\delta \lambda)^{n-1}}{(\gamma+\lambda-\gamma \lambda)(1-\delta)} .
$$

Because (19) is proportional to the left-hand side of (21), it follows that the objective of (P) is constant given (12) and (13). This implies the conclusion of the lemma.

Proof of Proposition 1: Part (i) is obvious. Part (ii) follows directly from Lemma 2.

Proof of Lemma 3: The first line of (14) is obtained by substitution of $u_{k}^{H}=\bar{u}^{H}$ into (6). Next, (20) can be rewritten as

$$
\sum_{k=0}^{n-1}\left(\begin{array}{c}
n-1 \\
k
\end{array}\right) \lambda^{k}(1-\lambda)^{n-k-1} u_{k+1}^{H}=\frac{1}{\gamma+\lambda-\gamma \lambda} \sum_{k=1}^{n}\left(\begin{array}{c}
n-1 \\
k-1
\end{array}\right) \lambda^{k-1}(1-\lambda)^{n-k} \bar{d}_{k}^{H}
$$

Because $\bar{d}_{0}^{H}=0$ and

$$
\left(\begin{array}{l}
n-1 \\
k-1
\end{array}\right)=\frac{k}{n}\left(\begin{array}{l}
n \\
k
\end{array}\right)
$$

the above equality becomes

$$
\sum_{k=0}^{n-1}\left(\begin{array}{c}
n-1 \\
k
\end{array}\right) \lambda^{k}(1-\lambda)^{n-k-1} u_{k+1}^{H}=\frac{1}{\lambda n(\gamma+\lambda-\gamma \lambda)} \sum_{k=0}^{n}\left(\begin{array}{l}
n \\
k
\end{array}\right) \lambda^{k-1}(1-\lambda)^{n-k} k \bar{d}_{k}^{H} .
$$

Substituting (22) into (19) implies the second line of (14).

Proof of Proposition 2: First, we prove part (i). Suppose $(\star)$ holds. Obviously, $\hat{Z}=Z^{S Q}$. By (10) and (11),

$$
Z^{S Q}-Z^{P A}=(1-\gamma) \sum_{k=0}^{n}\left(\begin{array}{l}
n \\
k
\end{array}\right) \lambda^{k}(1-\lambda)^{n-k} k\left(\bar{u}^{H}-\bar{d}_{k}^{H}\right)>0
$$

Next, we prove part (ii). Suppose $(\star)$ does not hold. It is obvious from (10) and (11) that $Z^{P A}>$ 
$Z^{S Q}$. By (11) and (14),

$$
\hat{Z}-Z^{P A}=(1-\gamma)\left(\frac{1}{\gamma+\lambda-\gamma \lambda}-1\right) \sum_{k=0}^{n}\left(\begin{array}{l}
n \\
k
\end{array}\right) \lambda^{k}(1-\lambda)^{n-k} k \bar{d}_{k}^{H}>0
$$

Proof of Proposition 3: Because $d_{k}^{H}(\alpha)=\bar{d}_{k}^{H}$ for any $\alpha$, for $\left\{u_{k}^{H}(\alpha), d_{k}^{H}(\alpha), d_{k}^{L}(\alpha)\right\}_{k=0}^{n}$ to implement an optimal solution $\left\{\hat{u}_{k}^{H}, \bar{d}_{k}^{H}, \hat{d}_{k}^{L}\right\}_{k=0}^{n}$ we only need $u_{k}^{H}(\alpha)$ to be equal to $\hat{u}_{k}^{H}$. First, suppose $(\star)$ holds. By Proposition 1 part (i), the unique optimal solution to $\left(\mathrm{MD}^{\prime}\right)$ corresponds to $\hat{u}_{k}^{H}=\bar{u}^{H}$. Because for any $k, u_{k}^{H}(\alpha)$ is strictly increasing in $\alpha, u_{k}^{H}(0)=\bar{d}_{k}^{H}$ and $u_{k}^{H}(1)=\bar{u}^{H}$, the unique $\hat{\alpha}$ so that $\left\{u_{k}^{H}(\hat{\alpha}), d_{k}^{H}(\hat{\alpha}), d_{k}^{L}(\hat{\alpha})\right\}_{k=0}^{n}$ implements the optimal solution is $\hat{\alpha}=1$. Next, suppose $(\star)$ does not hold. Let

$$
g(\alpha) \equiv \sum_{k=0}^{n-1}\left(\begin{array}{c}
n-1 \\
k
\end{array}\right) \lambda^{k}(1-\lambda)^{n-k-1}\left[\bar{d}_{k+1}^{H}-\gamma u_{k+1}^{H}(\alpha)-\frac{k}{n-k}\left(u_{k}^{H}(\alpha)-\bar{d}_{k}^{H}\right)\right] .
$$

Because $u_{k}^{H}(\alpha)$ is strictly increasing in $\alpha$ for any $k, g^{\prime}(\alpha)<0$. Applying $u_{k}^{H}(0)=\bar{d}_{k}^{H}$ yields

$$
\begin{aligned}
g(0) & =\sum_{k=0}^{n-1}\left(\begin{array}{c}
n-1 \\
k
\end{array}\right) \lambda^{k}(1-\lambda)^{n-k-1}\left[\bar{d}_{k+1}^{H}-\gamma \bar{d}_{k+1}^{H}-\frac{k}{n-k}\left(\bar{d}_{k}^{H}-\bar{d}_{k}^{H}\right)\right] \\
& =\sum_{k=0}^{n-1}\left(\begin{array}{c}
n-1 \\
k
\end{array}\right) \lambda^{k}(1-\lambda)^{n-k-1}(1-\gamma) \bar{d}_{k+1}^{H}>0 .
\end{aligned}
$$

Applying $u_{k}^{H}(1)=\bar{u}^{H}$ yields

$$
g(1)=\sum_{k=0}^{n-1}\left(\begin{array}{c}
n-1 \\
k
\end{array}\right) \lambda^{k}(1-\lambda)^{n-k-1}\left[\bar{d}_{k+1}^{H}-\gamma \bar{u}^{H}-\frac{k}{n-k}\left(\bar{u}^{H}-\bar{d}_{k}^{H}\right)\right]<0,
$$

where the inequality follows from the supposition that $(\star)$ does not hold (equivalently, $(8)$ does not hold). Hence, there exists a unique $\hat{\alpha} \in(0,1)$ such that $g(\hat{\alpha})=0$. Because $\left\{u_{k}^{H}(\hat{\alpha})\right\}_{k=0}^{n}$ satisfies (12) and (13), $\left\{u_{k}^{H}(\hat{\alpha}), \bar{d}_{k}^{H}\right\}_{k=0}^{n}$ is an optimal solution to $\left(\mathrm{MD}^{\prime}\right)$. 


\section{References}

Adar, E., And B. A. Huberman (2000): "Free Riding on Gnutella," First Monday, 5(10).

Adler, M., R. Kumar, K. Ross, D. Rubenstein, D. Turner, and D. D. Yao (2004): "Optimal Peer Selection in a Free-Market Peer-Resource Economy," in Workshop on Economics of Peer-to-Peer Systems.

Antoniadis, P., C. Courcoubetis, and R. Mason (2004): "Comparing Economic Incentives in Peer-to-Peer Networks," Computer Networks, 46(1), 113-146.

Antoniadis, P., C. Courcoubetis, and B. Strulo (2005): "Incentives for Content Availability in Memory-less Peer-to-Peer File Sharing Systems," ACM SIG on Ecommerce, 5(4), 11-20.

Buragohain, C., D. Agrawal, and S. Suri (2003): "A Game Theoretic Framework for Incentives in P2P Systems," in Proceedings of IEEE International Conference on Peer-to-Peer Computing.

Casadesus-Masanell, R., and A. Hervas-Drane (2007): "Peer-to-Peer File Sharing and the Market for Digital Information Goods," Working Paper, Harvard Business School.

Cisco Systems Inc (2007): "Cisco Visual Networking Index - Forecast and Methodology," http://www.cisco.com.

Feigenbaum, J., C. Papadimitriou, and S. Shenker (2005): "A BGP-based Mechanism for Lowest-Cost Routing," Distributed Computing, 18(1), 67-72.

Feigenbaum, J., and S. Shenker (2002): "Distributed Algorithmic Mechanism Design: Recent Results and Future Directions," in Proceedings of International Workshop on Discrete Algorithms and Methods for Mobile Computing and Communications.

Feldman, M., and J. Chuang (2005): "Overcoming Free-Riding Behavior in Peer-to-Peer Systems," ACM SIGecom Exchanges, 5(4), 41-50.

Feldman, M., C. Papadimitriou, J. Chuang, and I. Stoica (2006): "Free-Riding and Whitewashing in Peer-to-Peer Systems," IEEE Journal on Selected Areas in Communications, 24(5), 1010-1019.

Fudenberg, D., And J. Tirole (1991): Game Theory. Mit Press.

Golle, P., K. Leyton-Brown, I. Mironov, and M. Lillibridge (2001): "Incentives for Sharing in Peer-to-Peer Networks," in Proceedings of ACM Conference on Electronic Commerce.

Green, J. R., and J.-J. Laffont (1986): "Partially Verifiable Information and Mechanism Design," Review of Economic Studies, 53, 447-456.

Groves, T., And J. Ledyard (1977): "Optimal Allocation of Public Goods: A Solution to the "Free Rider" Problem," Econometrica, 45(4), 783-809. 
Gummadi, P. K., R. J. Dunn, S. Saroiu, S. D. Gribble, H. M. Levy, and J. Zahorjan (2003): "Measurement, Modeling, and Analysis of a Peer-to-Peer File-Sharing Workload," in Proceedings of ACM Symposium on Operating Systems Principles.

Habib, A., and J. Chuang (2006): "Service Differentiated Peer Selection: An Incentive Mechanism for Peer-to-Peer Media Streaming," IEEE Transactions on Multimedia, 8(3), 610-621.

Huang, Q., S. Huang, and C. Gao (2007): "A Differentiated Service Based Incentive Mechanism in P2P File-Sharing Systems," in Proceedings of IFIP International Conference on Network and Parellel Computing.

Hughes, D., G. Coulson, and J. Walkerdine (2005): "Free Riding on Gnutella Revisited: The Bell Tolls?," IEEE Distributed Systems Online, 6(6).

Kamvar, S. D., M. T. Schlosser, and H. Garcia-Molina (2003): "The EigenRep: Reputation Management in P2P Networks," in Proceedings of ACM International Conference on World Wide Web.

Krishnan, R., M. D. Smith, Z. Tang, and R. Telang (2004): "The Virtual Commons: Understanding Content Provision in Peer-to-Peer File Sharing Networks," Working Paper 2004-19, Carnegie Mellon Heinz School.

Krishnan, R., M. D. Smith, and R. Telang (2003): "The Economics of Peer-to-Peer Networks," Journal of Information Technology Theory and Applications, 5(3), 31-44.

Laffont, J.-J. (1987): "Incentives and the Allocation of Public Goods," in Handbook of Public Economics, ed. by A. J. Auerbach, and M. Feldstein, vol. 2, chap. 10, pp. 537-569. North Holland.

Livingstone, A. (2006): "A Bit of BitTorrent Bother," BBC Two.

Mas-Colell, A., M. Whinston, and J. R. Green (1995): Microeconomic Theory. Oxford University Press.

PAsick, A. (2004): "File Sharing Network Thrives Beneath the Radar," London Reuters.

Ranganathan, K., M. Ripeanu, A. Sarin, and I. Foster (2003): "To Share or not to Share: An Analysis of Incentives to Contribute in Collaborative File Sharing Environments," in Workshop on Economics of Peer-to-Peer Systems.

- (2004): "Incentive Mechanisms for Large Collaborative Resource Sharing," in Proceedings of International Conference on Peer-to-Peer Computing.

Roczniak, A., And A. E. SAdDIK (2005): "Impact of Incentive Mechanisms on Quality of Experience," in Proceedings of ACM International Conference on Multimedia.

Ruffo, G., and R. Shifanella (2007): "FairPeers: Efficient Profit Sharing in Fair Peer-to-Peer Market Places," Journal of Network and Systems Management, 15(3), 355-382. 
Saroiu, S., P. K. Gummadi, and G. D. Steven (2002): "A Measurment Study of Peer-to-Peer File Sharing Systems," in Proceedings of Multimedia Computing and Networking.

Schulze, H., And C. Mochalski (2007): "IPOQUE Internet Study," http://www.ipoque.com.

Shneidman, J., and D. C. Parkes (2003): "Rationality and Self-Interest in Peer to Peer Networks," in Proceedings of International Workshop on Peer-to-Peer Systems.

TAn, G., and S. A. Jarvis (2008): "A Payment-Based Incentive and Service Differentiation Scheme for Peer-to-Peer Streaming Broadcast," IEEE Transactions on Parallel and Distributed Systems, 19(7), 940-953.

Van der SchaAr, M., and D. S. Turaga (2008): "Stochastic Optimization for Content Sharing in P2P Systems," IEEE Transactions on Multimedia, 10(1), 132-144.

Yu, B., And M. P. Singh (2003): "Incentive Mechanisms for Agent-Based Peer-to-Peer Systems," in Proceedings of International Workshop on Agents and Peer-to-Peer Computing.

Zhang, Y., L. Lin, And J. Huai (2007): "Balancing Trust and Incentive in Peer-to-Peer Collaborative System," International Journal of Network Security, 5(1), 73-81. 